\title{
Disruption of intracardiac flow patterns in the newborn infant
}

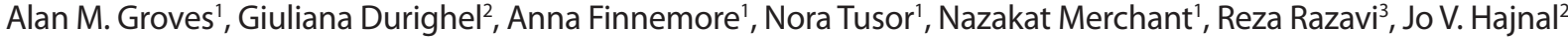 \\ and A. David Edwards'
}

INTRODUCTION: Consistent patterns of rotational intracardiac flow have been demonstrated in the healthy adult human heart. Intracardiac rotational flow patterns are hypothesized to assist in the maintenance of kinetic energy of inflowing blood, augmenting cardiac function. Newborn cardiac function is known to be suboptimal secondary to decreased receptor number and sympathetic innervation, increased afterload, and increased reliance on atrial contraction to support ventricular filling. Patterns of intracardiac flow in the newborn have not previously been examined.

RESULTS: Whereas 5 of the 13 infants studied showed significant evidence of rotational flow within the right atrium, 8 infants showed little or no rotational flow. Presence or absence of rotational flow was not related to gestational age, birth weight, postnatal age, atrial size, or image quality. Despite absence of intra-atrial rotational flow, atrioventricular valve flow into the left and right ventricles later in the cardiac cycle could be seen, suggesting that visualization techniques were adequate.

DISCUSSION: While further study is required to assess its exact consequences on cardiac mechanics and energetics, disruption to intracardiac flow patterns could be another contributor to the multifactorial sequence that produces newborn circulatory failure.

METHODS: We studied 13 newborn infants, using three-dimensional (3D) cardiac magnetic resonance phase-contrast imaging (spatial resolution $0.84 \mathrm{~mm}$, temporal resolution $22.6 \mathrm{~ms}$ ) performed without sedation/anesthesia.

$C^{2}$ ardiac magnetic resonance imaging (CMRI) studies in the healthy human adult have revealed consistent rotational flow patterns within the cardiac chambers (1-4). In the adult, right atrium inflowing blood from the superior vena cava (SVC) and inferior vena cava (IVC) are directed so as to avoid collision of flow and produce a consistent biphasic rotational pattern during ventricular systole and diastole $(1,5)$. It is hypothesized that these patterns maintain the momentum of inflowing bloodstreams by avoiding inefficient deceleration and reacceleration of blood, while the direction of rotational flow allows blood to "slingshot" into the right ventricle following tricuspid valve opening (5-7). There is growing interest in the complexities and impact on energetics of intracardiac flow patterns, (8) with much scope for further in vivo analysis $(9,10)$.
Circulatory failure is common in prematurely born infants and is associated with preventable mortality and long-term neurodevelopmental handicap. The circulation in the premature newborn is already impaired by decreased preload (11), poor inherent contractility $(12,13)$, high afterload (14) and shunting through the fetal pathways (15). Diastolic function is known to be altered in the newborn infant, with a significant decrease in ventricular compliance (16) as well as a significant decrease in passive ventricular filling and increased reliance on active diastolic filling during atrial systole $(17,18)$. Patterns of intracardiac flow in the functionally and structurally unique newborn human heart have not been studied. Any disruption to intracardiac flow patterns could be yet another contributor to this multifactorial sequence which produces manifest circulatory failure and could be causally related to the altered balance of passive and active diastolic ventricular filling.

During the transition from the fetal to the extrauterine circulation shortly after birth, aspects of the fetal circulation could cause significant disruption to intracardiac flow patterns. Prior studies examining circulatory physiology and pathophysiology of circulatory failure in the newborn have been hampered by the lack of sophistication in biomarkers of circulatory function (19). Arterial blood pressure, the most commonly measured marker of circulatory function, has a limited association with cardiac output (20). Ultrasound techniques have limited repeatability in the newborn population (21). Recently, CMRI techniques have been successfully applied to both the term and the preterm neonatal population $(19,22)$, without sedation or anesthesia and while maintaining cardiorespiratory and thermal stability (23). CMRI has been shown to have significantly increased repeatability over echocardiography in the neonatal population and also provides greater intricacy than echocardiography as it allows assessment of filling of the cardiac chambers as well as ejection fraction (19).

The aim of this study was to use 3D phase-contrast CMRI techniques to demonstrate the presence or absence of intracardiac rotational flow patterns in newborn human infants, with a small cohort of adults examined for comparison.

\section{RESULTS}

\section{Adult Imaging}

Whole-heart 3D phase-contrast imaging was performed in four healthy adult volunteers (one male, age range $27-38$ y).

\footnotetext{
'Department of Pediatrics, Hammersmith Hospital, Imperial College London, London, UK; '2Department of Imaging Sciences, Hammersmith Hospital, Imperial College London, London, UK; ${ }^{3}$ Division of Imaging Sciences, Guy's and St Thomas' National Health Service (NHS) Foundation Trust, King's College London, London, UK. Correspondence: Alan Groves (alan.groves@imperial.ac.uk) 
In all cases, image quality was assessed as 2 or 3 on a scale of $0-3$, see Methods. These four subjects had a mean (SD) heart rate of 62 (3.9) beats/min and mean (SD) vessel/chamber areas of IVC $459(96) \mathrm{mm}^{2}$, SVC $281(122) \mathrm{mm}^{2}$, right atrium $2,051(444) \mathrm{mm}^{2}$, and right ventricle $2,972(1,051) \mathrm{mm}^{2}$. At the resolution employed, this equated to voxels per vessel/ chamber of IVC 248 (52) voxels, SVC 152 (66) voxels, right atrium 1,109 (240) voxels, and right ventricle 1,607 (568) voxels. All four adults showed category 3 rotational flow (completing a full $360^{\circ}$ circle) within the right atrium. In all cases, rotational flow approximated the sagittal plane and was in a counterclockwise direction when viewed from the subjects left side (Figure 1a, Supplementary Video S1 online).

\section{Infant Imaging}

Whole-heart 3D phase-contrast imaging was performed in 14 newborns. No scan was complicated by significant desaturation, bradycardia, or hypothermia. No infant had evidence of structural congenital heart disease. In one case, the images were considered of insufficient quality for analysis. Demographics, image quality scores, heart rates, and assessments of intracardiac rotational flow patterns in the remaining 13 infants are shown in Table 1. These 13 infants had median (range) birth weight of $1,740(830-3,614) \mathrm{g}$ and birth gestation of $32(28-$ 41) wk, and 9 infants were male. At scan, postnatal age was 20 (1-37) d, weight was $1,890(1,020-3,614) \mathrm{g}$, and gestation was $35(30-41) \mathrm{wk}$.

These 13 subjects had a mean (SD) heart rate of 139 (21) beats/min; and mean (SD) vessel/chamber areas of IVC 46.3
(18.3) $\mathrm{mm}^{2}$, SVC $35.5(11.5) \mathrm{mm}^{2}$, right atrium $264(140) \mathrm{mm}^{2}$, and right ventricle $226(67) \mathrm{mm}^{2}$. At the resolution employed, this equated to voxels per vessel/chamber of IVC 66 (26) voxels, SVC 50 (16) voxels, right atrium 374 (198) voxels, and right ventricle 321 (96) voxels.

Three infants had category 3 (full $360^{\circ}$ ) rotational flow (Figure 1b, Supplementary Video S2 online), 2 had category 2 , and 6 had category 1 rotational flow. In 2 infants, there was no evidence of any rotational flow (Figure 1c, Supplementary Video S3 online).

Presence or absence of rotational flow was not associated with gestational age at birth $(P=0.24)$, birth weight $(P=0.17)$, postnatal age at scan $(P=0.70)$, gestation at scan $(P=0.45)$, weight at scan $(P=0.09)$, heart rate at scan $(P=0.52)$, or atrial area $(P=0.62)$.

Presence or absence of rotational flow did not appear to be associated with image acquisition quality - of the 6 infants with relatively poor image quality (grade 1), 3 had evidence of clear rotational flow, with or without a full $360^{\circ}$ cycle being seen. In addition, despite the limited image quality, flow in these infants could still be visualized clearly at the level of the mitral and tricuspid valves.

However, the presence or absence of rotational flow did appear to be associated with the course of IVC flow inside the right atrium. In 4 of 5 infants with rotational flow, flow from the IVC did not deviate from the foot-to-head direction within the right atrium (Figure 2a). By contrast, in 7 of 8 infants with no or minimal rotational flow, flow from the IVC was deflected anteriorly or to the left on entry to the right atrium (Figure 2b).

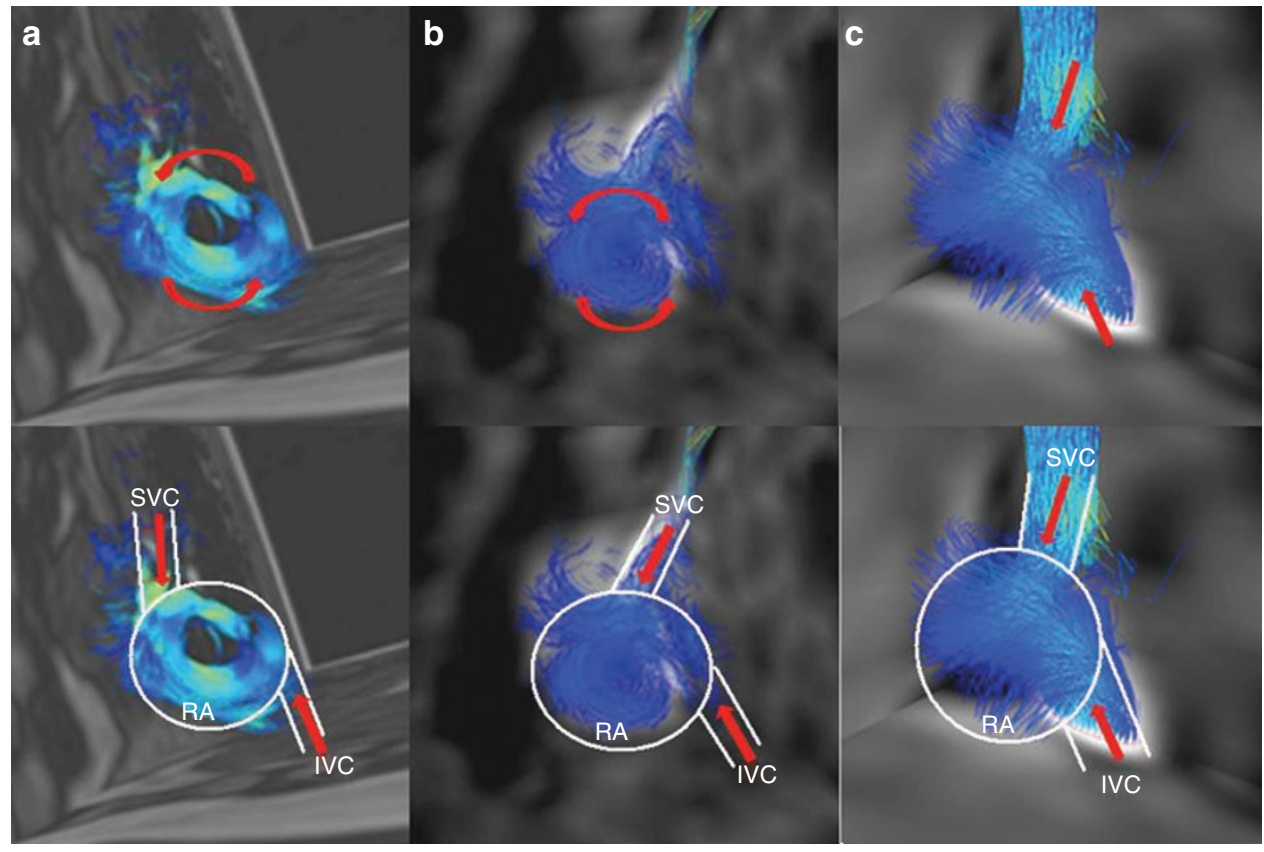

Figure 1. Presence and absence of rotational intracardiac flow patterns. Pathlines of inflowing superior and inferior vena caval blood in (a) a healthy adult volunteer, (b) a 1,020-g newborn (infant 1, Table 1), and (c) a healthy term newborn (infant 13, Table 1). In all cases, flow is visualized anteriorly and from the subject's left, and the lower panel includes a schematic of SVC, IVC, and RA structure for ease of interpretation. In cases (a) and (b), a full $360^{\circ}$ rotation is seen within the right atrium. In the infant shown in (c), there is no evidence of any rotational flow pattern within the right atrium. IVC, inferior vena cava; RA, right atrium; SVC, superior vena cava. 


\section{Articles | Grovesetal.}

Table 1. Demographics, image-quality scores, heart rates, and assessments of intracardiac flow patterns in 13 newborn infants

\begin{tabular}{|c|c|c|c|c|c|c|c|c|}
\hline Infant & $\begin{array}{l}\text { Gestational age } \\
\text { at scan (weeks) }\end{array}$ & $\begin{array}{l}\text { Postnatal } \\
\text { age (days) }\end{array}$ & $\begin{array}{l}\text { Weight at } \\
\operatorname{scan}(\mathrm{g})\end{array}$ & $\begin{array}{c}\text { Atrial area } \\
\left(\mathrm{mm}^{2} / \mathrm{kg}\right)\end{array}$ & Image quality & Heart rate & $\begin{array}{l}\text { Inferior vena cava } \\
\text { flow pattern }\end{array}$ & $\begin{array}{l}\text { Rotational } \\
\text { flow pattern }\end{array}$ \\
\hline 1 & 30.86 & 10 & 1,020 & 110 & 1 & 142 & Straight & 3 \\
\hline 2 & 33.57 & 36 & 1,840 & 85 & 3 & 170 & Deflected & 1 \\
\hline 3 & 33.57 & 36 & 2,070 & 153 & 2 & 175 & Straight & 3 \\
\hline 4 & 33.71 & 37 & 1,100 & 88 & 1 & 152 & Deflected & 2 \\
\hline 5 & 33.71 & 37 & 1,545 & 108 & 2 & 159 & Deflected & 1 \\
\hline 6 & 35.14 & 18 & 1,890 & 110 & 1 & 139 & Deflected & 1 \\
\hline 7 & 35.14 & 2 & 2,054 & 172 & 2 & 112 & Straight & 3 \\
\hline 8 & 35.57 & 24 & 1,990 & 152 & 1 & 135 & Deflected & 1 \\
\hline 9 & 36.57 & 20 & 1,875 & 101 & 1 & 140 & Straight & 2 \\
\hline 10 & 36.57 & 20 & 1,880 & 115 & 2 & 138 & Deflected & 0 \\
\hline 11 & 36.71 & 3 & 3,086 & 84 & 3 & 122 & Deflected & 1 \\
\hline 12 & 37.14 & 1 & 3,598 & 139 & 1 & 113 & Straight & 1 \\
\hline 13 & 41.43 & 2 & 3,614 & 153 & 3 & 113 & Deflected & 0 \\
\hline
\end{tabular}

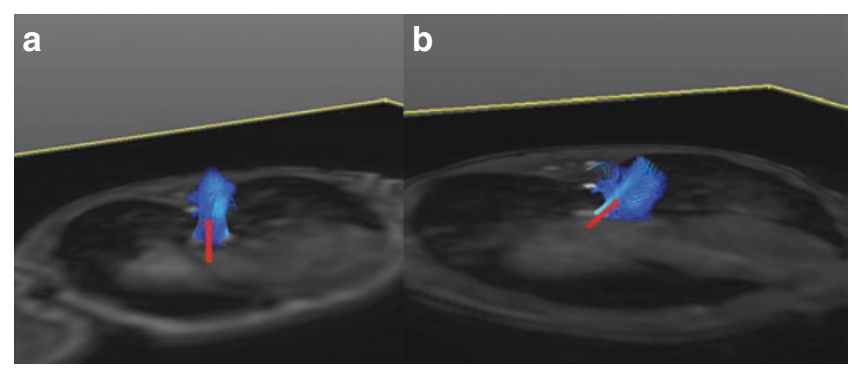

Figure 2. Direction of blood flow on entry to the right atrium. Pathlines of inflowing inferior vena caval blood in (a) a newborn with direct flow into the right atrium and (b) a newborn with IVC flow deflected anteriorly and to the left. Flow is visualized anteriorly and from the subject's right.

\section{DISCUSSION}

This study demonstrates that rotational intracardiac flow patterns that are consistently seen in healthy adults are not present in some newborn infants. Although it is possible that we have failed to visualize rotational flow patterns that formed in the subjects studied, this is relatively unlikely.

The temporal resolution of our sequence was $22.6 \mathrm{~ms}$, which was sufficient for the robust construction of 20 phases/cardiac cycle, even with the relatively high heart rates seen in our subjects. This is equivalent to the temporal resolution used in other studies (1). In addition, when present, rotational flow patterns are visible over multiple phases of the cardiac cycle (1).

The spatial resolution used in our study is somewhat more limited. However, a voxel size of $<1 \mathrm{~mm}$ displayed a mean of 374 voxels within the right atria of infants studied. While this equates to only around a third of the voxel coverage seen in adults, in our study it is equivalent to the coverage employed to successfully demonstrate rotational flow patterns in the ascending aorta in adult subjects (24). In addition, there was no association between atrial size and presence or absence of rotational flow.

Given that our images are averaged over multiple phases of the respiratory cycle, it is theoretically possible that rotational flow patterns are seen in some infants in certain respiratory phases but not in others or that respiratory motion degraded the image quality. However, rotational patterns were consistently seen in adult subjects using the same free-breathing technique, and neonatal CMRI image quality appears to be less vulnerable to respiratory-motion artifact than adult imaging (19). Presence or absence of rotational flow was not associated with image-acquisition quality, flow could be reliably traced through to the phase of ventricular filling, and an unambiguous pattern of nonrotational flow was seen across a range of infant weights.

As previously stated, rotational flow patterns may increase cardiac efficiency by maintaining kinetic energy and direction of flow $(5,7)$. It has been noted that the looped cardiac structure and the atrioventricular relationship predispose to rotational intracardiac patterns in humans and that looped cardiac structures are not seen in less active species such as snails (1). Loss of rotational flow patterns has been postulated to impact on cardiac efficiency in adult pathologies such as postmyocardial infarct (5) and in dilated cardiomyopathy (2). Studies in subjects with congenital heart disease have suggested that the geometry of inflowing blood to the total cavopulmonary connection has a significant impact on flow efficiency (25). Although the potential momentum-conserving impact of rotational flow patterns in the adult ventricle has not always been confirmed by computational modeling studies (26), these techniques have not yet been applied to the atria. Any momentumconserving effect of rotational flow in the ventricles might be minor compared with the energy injected during ventricular systole, but this is not the case during ventricular diastole. In ventricular diastole, passive filling is vital and relatively minor decreases in the kinetic energy of atrial blood may significantly impair ventricular filling.

Cardiac function in the newborn is known to be significantly different from that in older children and adults. Newborns have decreased capacity for myocardial force generation $(12,13)$, have decreased sympathetic innervation (27) and receptor number (28), and are forced to pump against high afterload related to the sudden clamping off of the lowresistance placental circulation (14). Doppler echocardiography studies have demonstrated that neonatal ventricular 
filling is heavily reliant on atrial contraction, in contrast to the adult pattern where passive ventricular filling predominates. Newborn infants are also vulnerable to diastolic dysfunction because of their high heart rates $(17,29)$, low ventricular compliance $(30,31)$, and low preload associated with rapid increase in capacitance of the pulmonary vasculature with the first few postnatal breaths (11). In addition, studies of fetal and neonatal myocardial structure have shown altered subtypes of collagen (30) and titin molecules (32). These variations are associated with decreased efficacy of ventricular twist in systole and an impairment of recoil with potential effects on diastolic filling velocity (33).

The loss of rotational intracardiac flow patterns may be yet another factor contributing to the risk of circulatory dysfunction in the newborn and could in fact be causally related to the altered balance of passive and active ventricular filling, although quantitative analysis of both rotational flow pattern and diastolic function are clearly necessary to test this hypothesis. If confirmed, diastolic dysfunction related to absent rotational patterns may leave infants particularly vulnerable to impact of low preload, for example, during sepsis, and to the impact of tachycardia (worsened by the use of inotropic agents) when the time available for ventricular filling is already limited.

We have not yet been able to define the mechanism(s) of disruption of rotational flow in the newborn. However, the association between absence of rotational flow and deflection of blood from the IVC on entry to the right atrium is noteworthy. The eustachian valve functions in the fetal circulation to direct blood from the IVC across the foramen ovale into the left atrium and remains prominent in the neonatal period, projecting, on average, $5 \mathrm{~mm}$ into the right atrium (34). By preventing blood from the IVC flowing vertically up the posterior wall of the right atrium (as seen in adults), the eustachian valve could be deflecting blood from out of the adult rotational flow path. The eustachian valve is too thin to be visible at current spatial resolution with CMRI. Although shunt across the foramen ovale is right to left in the fetal circulation, it rapidly becomes predominantly left to right in the postnatal circulation, and the foramen ovale can remain patent for some weeks (35), with shunting tending to be more significant in preterm infants (36). Blood flowing from the left atrium into the right atrium could therefore be disrupting rotational flow and would not be visualized by our methodology.

This study has significant limitations. To date, only small numbers of infants have been studied, so a reliable estimate of the frequency with which rotational flow is disrupted cannot be made. Our analysis of rotational flow is qualitative rather than quantitative (2), and we have not yet linked the presence or absence of rotational flow to ventricular diastolic dysfunction.

\section{Conclusion}

3D CMRI techniques suggest that intracardiac rotational flow patterns may be disrupted in the newborn infant. Further study is required to assess potential impact on preservation of momentum and diastolic function, but disruption of intracardiac flow patterns may be a further risk factor for circulatory dysfunction in this vulnerable population.

\section{METHODS}

MR imaging was approved by the Hammersmith Hospitals Trust Research Ethics Committee, and written subject/parental consent was obtained for all studies.

\section{Adult Scan Acquisition}

Adult scans were performed using a 1.5 tesla Philips (Best, Netherlands) Achieva MRI scanner with an eight-channel receiver coil. The whole heart was imaged in a single acquisition in the axial or sagittal plane using a 3D phase-contrast pulse sequence. All sequences were acquired during free breathing, without the use of respiratory-compensation techniques and with retrospective cardiac gating. Acquisition parameters were repetition time (TR), $5.4 \mathrm{~ms}$; echo time (TE), $2.52 \mathrm{~ms}$; flip angle, $15^{\circ}$; matrix, $256 \mathrm{~mm}$; acquired voxel size, $3 \mathrm{~mm}$; reconstructed voxel size, $1.36 \mathrm{~mm}$; slice thickness, $3 \mathrm{~mm}$; number of slices, 40 ; temporal resolution, $17.2 \mathrm{~ms}$; number of cardiac phases, 20; number of excitations (NSA), 1. Velocity (VENC) was encoded at $150 \mathrm{~cm} / \mathrm{s}$ in all three directions. Mean (SD) scan duration for 3D flow acquisition in adults was $564(74) \mathrm{s}$.

\section{Infant Scan Acquisition}

Infant scans were performed using a 3.0 tesla Philips (Best, Netherlands) Achieva MRI scanner with a Flex-M two-channel surface receiver coil placed on each side of the chest wall. Infants studied were inpatient in either the neonatal intensive care unit or the postnatal ward of Queen Charlotte's and Chelsea Hospital and were scanned solely for cardiac imaging research. All infants were stable at the time of imaging, tolerating full enteral feeds and receiving lowflow supplemental oxygen or no respiratory support as clinically indicated. No infant was receiving inotropic support. Infants were fed and allowed to fall into natural sleep without sedation or anesthesia, then laid in an MRI-compatible cradle with ear protection, pulse oximetry, and vector electrocardiogram monitoring. All sequences were acquired free breathing, with no respiratory compensation techniques used and with retrospective ECG gating. A pediatrician was present throughout to monitor clinical stability.

The whole heart was imaged in a single acquisition in the axial plane using a $3 \mathrm{D}$ phase-contrast pulse sequence. Acquisition parameters were TR, $5.6 \mathrm{~ms}$; TE, $3.42 \mathrm{~ms}$; flip angle, $15^{\circ}$; matrix, $224 \mathrm{~mm}$; acquired voxel size, $2.5 \mathrm{~mm}$; reconstructed voxel size, $0.84 \mathrm{~mm}$; slice thickness, $2 \mathrm{~mm}$; number of slices, 20; temporal resolution, $22.6 \mathrm{~ms}$; number of cardiac phases, 20; NSA, 1; and velocity (VENC) was encoded at $150 \mathrm{~cm} / \mathrm{s}$ in all three directions. Mean (SD) scan duration for 3D flow acquisition in infants was 432 (93) s.

Atrial area was taken from a steady-state free-procession fourchamber image (19), with the atrial endocardial border traced from the last frame before tricuspid valve opening (37).

\section{Flow Visualization}

Offline flow visualization of both adult and newborn acquisitions was performed using GTFlow software (release 1.4.13, GyroTools, Switzerland). Prior to flow visualization, magnitude and phase images of each acquisition were assessed for image quality by a single observer (A.M.G.) using a subjective scale where $0=$ unsuitable for analysis; 1 = acceptable for analysis, significant artifacts present; 2 = good image quality, almost no artifacts; and 3 = excellent image quality, no artifacts (Figure 3). To analyze flow within the right atrium, the SVC and IVC were defined on appropriate axial slices immediately upstream to the vessels entering the right atrium. Vessel border was defined using a semiautomatic contour-detection technique with freehand correction where necessary.

Pathlines to visualize flow were positioned to originate from the SVC and IVC from the axial slices immediately upstream to entering the right atrium. To maximize visualization, pathlines were initiated at end diastole and repeated twice within the next 2 of the 20 cardiac 


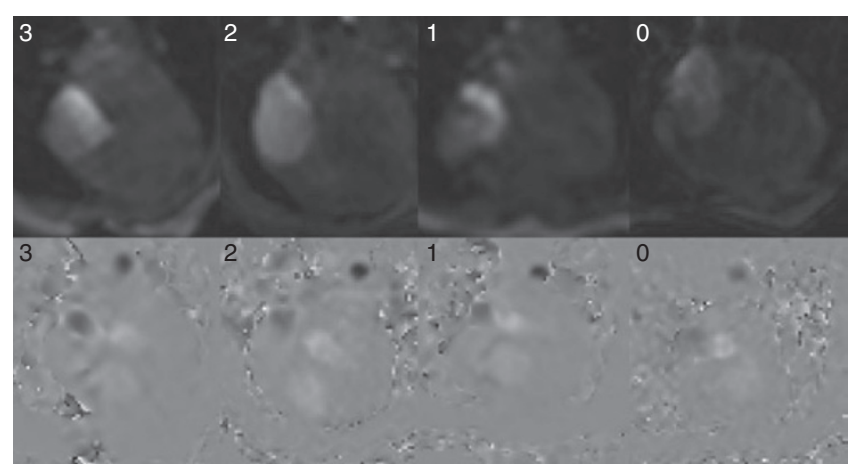

Figure 3. Assessments of image quality for magnitude and phase images. 0 , Unsuitable for analysis; 1 , acceptable for analysis, significant artifacts present; 2 , good image quality, almost no artifacts; 3 , excellent image quality, no artifacts.

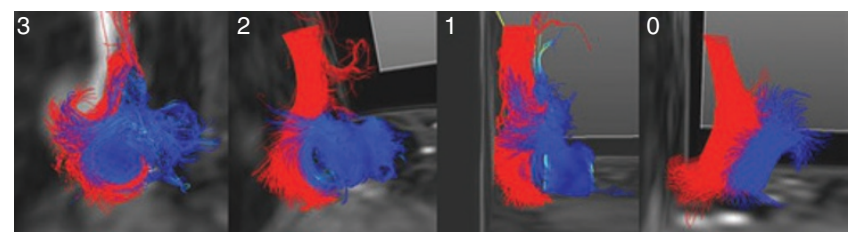

Figure 4. Categorization of intracardiac rotational flow patterns within the right atrium. 0 , No evidence of any rotational flow; 1 , minimal rotational flow; 2 , clear rotational flow, but not completing a full $360^{\circ}$ circle; 3 , rotational flow completing a full $360^{\circ}$ circle within the right atrium.

phases acquired. To obtain a dense sampling of the measured flow pattern, pathlines were depicted at triple density. This enables clear visualization despite the necessarily limited resolution in a newborn heart. The trace length (controlling the display of the length of the pathlines) was set to a quarter of the duration of the cardiac cycle. Flow pattern was investigated from all directions and at multiple time points throughout ventricular systole. Intracardiac rotational flow patterns within the right atrium were categorized as $0=$ no evidence of any rotational flow; $1=$ minimal rotational flow; $2=$ clear rotational flow, but not completing a full $360^{\circ}$ circle; and $3=$ rotational flow completing a full $360^{\circ}$ circle within the right atrium (Figure 4).

\section{Statistical Analysis}

Demographics of the infants studied are presented as median and range. Differences in presence or absence of rotational flow with demographic factors and heart rate were assessed using an unpaired two-tail Student's $t$ test. A $P$ value of $<0.05$ was taken as statistically significant.

\section{SUPPLEMENTARY MATERIAL}

Supplementary material is linked to the online version of the paper at http:// www.nature.com/pr

\section{ACKNOWLEDGMENTS}

We are grateful to the families who consented to take part in the study and to the nursing and medical staff members who participated in caring for the infants. We thank the staff of the Imaging Sciences Department of Imperial College London for their support.

\section{STATEMENT OF FINANCIAL SUPPORT}

This study was supported by the Garfield Weston Foundation, the Medical Research Council, and the Imperial College Comprehensive Biomedical Research Centre.

Disclosure: R.R., J.V.H., and A.D.E receive investigator-led research grant support from Philips.

\section{REFERENCES}

1. Kilner PJ, Yang GZ, Wilkes AJ, Mohiaddin RH, Firmin DN, Yacoub $\mathrm{MH}$. Asymmetric redirection of flow through the heart. Nature 2000;404:759-61.

2. Bolger AF, Heiberg E, Karlsson M, et al. Transit of blood flow through the human left ventricle mapped by cardiovascular magnetic resonance. J Cardiovasc Magn Reson 2007;9:741-7.

3. Kim WY, Walker PG, Pedersen EM, et al. Left ventricular blood flow patterns in normal subjects: a quantitative analysis by three-dimensional magnetic resonance velocity mapping. J Am Coll Cardiol 1995;26: 224-38.

4. Fyrenius A, Wigström L, Ebbers T, Karlsson M, Engvall J, Bolger AF. Three dimensional flow in the human left atrium. Heart 2001;86: 448-55.

5. Yang GZ, Merrifield R, Masood S, Kilner PJ. Flow and myocardial interaction: an imaging perspective. Philos Trans R Soc Lond, B, Biol Sci 2007;362:1329-41.

6. Kilner PJ. Postulated functional advantages of a looped as opposed to a linearly arranged heart [letter]. Am J Physiol Heart Circ Physiol 2010;298:H726; author reply H727.

7. Kilner PJ, Yang GZ, Firmin DN. Morphodynamics of flow through sinuous curvatures of the heart. Biorheology 2002;39:409-17.

8. Carlhäll CJ, Bolger A. Passing strange: flow in the failing ventricle. Circ Heart Fail 2010;3:326-31.

9. Markl M, Kilner PJ, Ebbers T. Comprehensive $4 \mathrm{D}$ velocity mapping of the heart and great vessels by cardiovascular magnetic resonance. J Cardiovasc Magn Reson 2011;13(1):7.

10. Markl M, Geiger J, Stiller B, Arnold R. Impaired continuity of flow in congenital heart disease with single ventricle physiology. Interact Cardiovasc Thorac Surg 2011;12:87-90.

11. Hong YM, Choi JY. Pulmonary venous flow from fetal to neonatal period. Early Hum Dev 2000;57:95-103.

12. Gill AB, Weindling AM. Echocardiographic assessment of cardiac function in shocked very low birthweight infants. Arch Dis Child 1993;68(1 Spec No): $17-21$.

13. Takahashi Y, Harada K, Kishkurno S, Arai H, Ishida A, Takada G. Postnatal left ventricular contractility in very low birth weight infants. Pediatr Cardiol 1997;18:112-7.

14. Groves AM, Kuschel CA, Knight DB, Skinner JR. Relationship between blood pressure and blood flow in newborn preterm infants. Arch Dis Child Fetal Neonatal Ed 2008;93:F29-32.

15. Groves AM, Kuschel CA, Knight DB, Skinner JR. Does retrograde diastolic flow in the descending aorta signify impaired systemic perfusion in preterm infants? Pediatr Res 2008;63:89-94.

16. Iwashima S, Seguchi M, Ohzeki T. Left ventricular diastolic performance in neonates. Circ J 2005;69:1094-8.

17. Hiarada K, Orino T, Yasuoka K, Tamura M, Takada G. Tissue doppler imaging of left and right ventricles in normal children. Tohoku J Exp Med 2000;191:21-9.

18. Mourani PM, Ivy DD, Rosenberg AA, Fagan TE, Abman SH. Left ventricular diastolic dysfunction in bronchopulmonary dysplasia. J Pediatr 2008;152:291-3.

19. Groves AM, Chiesa G, Durighel G, et al. Functional cardiac MRI in preterm and term newborns. Arch Dis Child Fetal Neonatal Ed 2011;96:F86-91.

20. Osborn DA, Evans N, Kluckow M. Clinical detection of low upper body blood flow in very premature infants using blood pressure, capillary refill time, and central-peripheral temperature difference. Arch Dis Child Fetal Neonatal Ed 2004;89:F168-73.

21. Chew MS, Poelaert J. Accuracy and repeatability of pediatric cardiac output measurement using Doppler: 20-year review of the literature. Intensive Care Med 2003;29:1889-94.

22. Foran AM, Fitzpatrick JA, Allsop J, et al. Three-tesla cardiac magnetic resonance imaging for preterm infants. Pediatrics 2007;120:78-83.

23. Merchant N, Groves A, Larkman DJ, et al. A patient care system for early 3.0 Tesla magnetic resonance imaging of very low birth weight infants. Early Hum Dev 2009;85:779-83. 
24. Markl M, Draney MT, Hope MD, et al. Time-resolved 3-dimensional velocity mapping in the thoracic aorta: visualization of 3-directional blood flow patterns in healthy volunteers and patients. J Comput Assist Tomogr 2004;28:459-68.

25. Sharma S, Ensley AE, Hopkins $\mathrm{K}$, et al. In vivo flow dynamics of the total cavopulmonary connection from three-dimensional multislice magnetic resonance imaging. Ann Thorac Surg 2001;71:889-98.

26. Watanabe H, Sugiura S, Hisada T. The looped heart does not save energy by maintaining the momentum of blood flowing in the ventricle. Am J Physiol Heart Circ Physiol 2008;294:H2191-6.

27. Barrington KJ. Hypotension and shock in the preterm infant. Semin Fetal Neonatal Med 2008;13:16-23.

28. Whitsett JA, Noguchi A, Moore JJ. Developmental aspects of alpha- and beta-adrenergic receptors. Semin Perinatol 1982;6:125-41.

29. Sarnari R, Kamal RY, Friedberg MK, Silverman NH. Doppler assessment of the ratio of the systolic to diastolic duration in normal children: relation to heart rate, age and body surface area. J Am Soc Echocardiogr 2009;22:928-32.

30. Marijianowski MM, van der Loos CM, Mohrschladt MF, Becker AE. The neonatal heart has a relatively high content of total collagen and type I collagen, a condition that may explain the less compliant state. J Am Coll Cardiol 1994;23:1204-8.
31. Romero T, Covell J, Friedman WF. A comparison of pressure-volume relations of the fetal, newborn, and adult heart. Am J Physiol 1972;222:1285-90.

32. Lahmers S, Wu Y, Call DR, Labeit S, Granzier H. Developmental control of titin isoform expression and passive stiffness in fetal and neonatal myocardium. Circ Res 2004;94:505-13.

33. Sengupta PP, Tajik AJ, Chandrasekaran K, Khandheria BK. Twist mechanics of the left ventricle: principles and application. JACC Cardiovasc Imaging 2008;1:366-76.

34. Limacher MC, Gutgesell HP, Vick GW, Cohen MH, Huhta JH. Echocardiographic anatomy of the eustachian valve. Am J Cardiol 1986;57: 363-5.

35. Connuck D, Sun JP, Super DM, et al. Incidence of patent ductus arteriosus and patent foramen ovale in normal infants. Am J Cardiol 2002;89: 244-7.

36. Evans N, Iyer P. Incompetence of the foramen ovale in preterm infants supported by mechanical ventilation. J Pediatr 1994;125(5 Pt 1): 786-92.

37. Whitlock M, Garg A, Gelow J, Jacobson T, Broberg C. Comparison of left and right atrial volume by echocardiography versus cardiac magnetic resonance imaging using the area-length method. Am J Cardiol 2010;106:1345-50. 